\title{
X-ray observations of two candidate symbiotic binaries in the galactic bulge
}

\author{
Joshua Wetuski, ${ }^{\star \star}$ R. I. Hynes, ${ }^{1 \star}$ T. J. Maccarone, ${ }^{2}$ C. Heinke ${ }^{\circledR},{ }^{3}$ M. A. P. Torres,${ }^{4,5}$ P. G. Jonker, ${ }^{6,7}$ \\ Christopher T. Britt, ${ }^{8}$ D. Steeghs ${ }^{9}$ and G. Nelemans ${ }^{6,7,10}$ \\ ${ }^{1}$ Department of Physics \& Astronomy, Louisiana State University, 202 Nicholoson Hall, Baton Rouge, LA 70803, USA \\ ${ }^{2}$ Department of Physics, Texas Tech University, Science Building, Box 41051, Lubbock, TX 79409-1051, USA \\ ${ }^{3}$ Department of Physics, University of Alberta, CCLS 4-183, Edmonton, AB T6G 2E1, Canada \\ ${ }^{4}$ Instituto de Astrofísica de Canarias (IAC), Vía Láctea s/n, E-38205 La Laguna, Santa Cruz de Tenerife, Spain \\ ${ }^{5}$ Departamento de Astrofisica, Universidad de La Laguna, E-38206 La Laguna, Santa Cruz de Tenerife, Spain \\ ${ }^{6}$ Department of Astrophysics/IMAPP, Radboud University, P.O. Box 9010, NL-6500 GL Nijmegen, the Netherlands \\ ${ }^{7}$ SRON, Netherlands Institute for Space Research, Sorbonnelaan 2, NL-3584 CA Utrecht, the Netherlands \\ ${ }^{8}$ Space Telescope Science Institute, 3700 San Martin Dr, Baltimore, MD 21218, USA \\ ${ }^{9}$ Department of Physics, University of Warwick, Coventry CV4 7AL, UK \\ ${ }^{10}$ Institute of Astronomy, KU Leuven, Celestijnenlaan 200D, B-3001 Leuven, Belgium
}

Accepted 2021 July 16. Received 2021 June 30; in original form 2021 April 19

\begin{abstract}
This paper analyses X-ray observations by the Chandra X-ray Observatory of CXOGBS J174614.3-321949 (CXB3) and CXOGBS J173620.2-293338 (CX332), two symbiotic binary star candidates identified by the Galactic Bulge Survey. Using new Chandra observations, we improved their X-ray positional uncertainties to 0.24 and 0.92 arcsec, respectively, confidently associating them with single optical counterparts. In particular, new observations of symbiotic X-ray candidate CX332 further solidify confidence in its coincidence with a carbon star. We demonstrate X-ray variability in both targets with a more recent observation of CX332 showing a decrease in brightness by a factor of 30, while CXB3 observations show it usually in a quiescent state with a factor-of-6 flare-like event in the final observations. In a combined spectral fit for CXB3, we find an $N_{\mathrm{H}}$ value of $\sim(2-3) \times 10^{22} \mathrm{~cm}^{-2}$ with a $\Gamma$ value of $1.5_{-0.2}^{+0.2}$ for a power-law fit and $k T 10.6_{-2.9}^{+5.7} \mathrm{keV}$ for an APEC fit and an estimated luminosity of $\sim 8.4 \times 10^{32} \mathrm{erg} \mathrm{s}^{-1}$. Spectra of CXB3 would be consistent with thermal emission as seen in white dwarf symbiotic systems, but the high X-ray luminosity in the light curve is more typically seen in symbiotic X-ray binary systems. Optical spectra of both objects taken with Gemini GMOS indicate CXB3 as containing an M-type star and CX332 having a carbon star counterpart. Both targets show at most marginal evidence of $\mathrm{H} \alpha$ emission favouring a symbiotic X-ray binary interpretation for both sources, though we cannot rule out a white dwarf for either case.
\end{abstract}

Key words: binaries: spectroscopic - binaries: symbiotic-X-rays: binaries.

\section{INTRODUCTION}

Symbiotic stars are a type of binary system that are interacting through wind accretion by transferring mass from a red giant, referred to as the donor star, on to a compact object, designated as the accretor. This process can be observed and identified by key defining characteristics in the red giant's emission line spectrum (Mikołajewska 2007). They typically display a red continuum with absorption like that of late-type giants and a blue continuum with bright emission lines that are common in accreting binary systems, though the blue continuum is often not directly seen as the optical is overwhelmed by the counterpart giant's light (Kenyon 1986).

The most common type of symbiotic binary systems are known to contain white dwarfs (WDs) as their compact accreting objects. These systems can be divided into different types based on their

^E-mail: jwetus1@1su.edu (JW); rih@phys.1su.edu (RIH) observed X-ray spectra (Muerset, Wolff \& Jordan 1997; Luna et al. 2013). The $\alpha$-type systems emit supersoft X-rays with photons having energies below $1 \mathrm{keV}$ with an origin likely coming from the surface of the WD burning in a quasi-steady state (Orio et al. 2007). Systems emitting soft $\mathrm{X}$-rays, peaking under $2 \mathrm{keV}$, are designated as $\beta$-type systems with emission likely originating from wind from the WD colliding with wind from the red giant companion. Systems emitting highly absorbed hard X-rays, peaking above $3 \mathrm{keV}$, are $\delta$-type systems with emission possibly originating in the boundary layer. There also exist $\beta / \delta$-type systems that are likely displaying both behaviours suggested in the $\beta$-type and $\delta$-type systems (Luna et al. 2013).

A smaller population of symbiotic binaries known as symbiotic $\mathrm{X}$-ray binaries (SyXBs) host either a neutron star or black hole as the accreting object (Masetti et al. 2006). The donor star in these systems is usually an M-type giant and they generally have hard Xray spectra with broad hard slopes similar to those of X-ray pulsars seen in high-mass X-ray binary systems (Enoto et al. 2014). These 
systems are designated as $\gamma$-type systems with X-ray origins likely due to optically thick Comptonized plasma (Masetti et al. 2007b; Marcu et al. 2011).

The Galactic population of these SyXBs has been estimated to be somewhere between a few hundred and a thousand with currently only 13 likely candidates (Lü et al. 2012; Yungelson, Kuranov \& Postnov 2019) with GX 1+4 being the most studied to date (Chakrabarty \& Roche 1997). Characteristics of studied SyXBs show inferred X-ray luminosity ranges of $10^{32}-10^{34} \mathrm{erg} \mathrm{s}^{-1}$ with both very hard and highly variable X-ray spectra, though GX $1+4$ is exceptionally bright with an X-ray luminosity of $10^{37} \mathrm{erg} \mathrm{s}^{-1}$ (Masetti et al. 2002, 2006).

The SyXBs, as systems with long orbital periods, are likely to have formed with relatively small velocity kicks, as large kicks would have to have been exceptionally finely tuned for the systems to remain bound. These systems may then disproportionately have electroncapture supernova products as their neutron stars, and thus represent good cases for estimating the masses, and hence constraining the binding energies of these lower mass neutron stars. WD symbiotics also appear to be possible progenitors of Type Ia supernovae. It has been discussed that they are ancestors of both the favoured double degenerate WD progenitors (Maoz, Mannucci \& Nelemans 2014) and single degenerate WD channels in their own right (Tutukov \& Yungelson 1979; Munari \& Renzini 1992; Kenyon et al. 1993).

It is difficult to confirm the identities of SyXBs as their optical spectra usually lack emission lines that make it hard to identify them with optical spectroscopy alone. There may also be a population of WD symbiotic binaries that show weak or no emission lines that is larger than the strong-line population (Mukai et al. 2016). X-ray surveys could possibly be used to detect these targets by finding $\mathrm{X}$-ray emission from neutron stars associated with giant stars that would go unnoticed in optical surveys.

There are two candidate SyXBs that have been identified in the Galactic Bulge Survey (GBS; Jonker et al. 2011, 2014). The GBS is a $0.3-8.0-\mathrm{keV}$ multiwavelength $\mathrm{X}$-ray survey performed with Chandra's ACIS-I camera specifically optimized to identify low-luminosity neutron star X-ray binaries in the bulge. CXOGBS J173620.2-293338, also designated as CX332, has J2000 equatorial coordinates $\alpha=17^{\mathrm{h}} 36^{\mathrm{m}} 20^{\mathrm{s}} .20, \delta=-29^{\circ} 33^{\prime} 39^{\prime \prime} 0$ and Galactic coordinates are $l=358.3978^{\circ}, b=-1.3869^{\circ}$. It is coincident with the very red star 2MASS J17362020-2933389, which has been classified as an asymptotic giant branch (AGB)-type carbon star (Hynes et al. 2014). CX332 displayed X-ray hardness and luminosity typical of that seen in other SyXBs, and its counterpart showed a lack of optical emission lines. These observations of CX332 fit well in a class of other SyXBs suggesting it is a candidate, but could not be confirmed due to the small number of photons detected off-axis yielding a poor localization with an error circle radius of 7.85 arcsec.

The second SyXB candidate is CXOGBS J174614.3-321949, with the GBS catalogue designation CXB3, having J2000 equatorial coordinates $\alpha=17^{\mathrm{h}} 46^{\mathrm{m}} 14.34, \delta=-32^{\circ} 19^{\mathrm{s}} 49^{\prime} .0$ and Galactic coordinates $l=357.1701^{\circ}, b=-1.8794^{\circ}$. This target was discovered in the field of view of Chandra X-ray Observatory (CXO) observations of $\mathrm{H} 1743-322$, a candidate black hole that went into outburst in 2003 (Corbel et al. 2005). CXB3 was among the brightest X-ray sources in the GBS and its optical counterpart has also been classified as a possible AGB or red giant branch star; therefore, it has been suggested that CXB3 is also a potential SyXB candidate (Jonker et al. 2014).

In this work, we examine the properties of CXB3 and CX332 to determine whether their compact objects are WDs or neutron stars and confirm whether these two objects are symbiotic binary systems.
Table 1. Log of CXO observations.

\begin{tabular}{ccccc}
\hline Object & Instrument & ObsID & Date & Exposure $(\mathrm{ks})$ \\
\hline CX332 & ACIS-I & 8693 & $05 / 19 / 2009$ & 2.0 \\
& ACIS-I & 15799 & $02 / 02 / 2016$ & 25.0 \\
CXB3 & ACIS-S & 4565 & $02 / 18 / 2005$ & 25.0 \\
& ACIS-S & 4566 & $03 / 29 / 2005$ & 30.0 \\
& ACIS-S & 4567 & $03 / 29 / 2005$ & 40.0 \\
& ACIS-S & 8987 & $03 / 03 / 2009$ & 7.0 \\
& ACIS-S & 8988 & $03 / 09 / 2009$ & 15.0 \\
& ACIS-S & 8989 & $03 / 17 / 2009$ & 22.5 \\
& ACIS-S & 8990 & $03 / 25 / 2009$ & 22.5 \\
& ACIS-S & 9833 & $03 / 17 / 2009$ & 12.5 \\
& ACIS-S & 9837 & $03 / 25 / 2009$ & 21.0 \\
& ACIS-S & 9838 & $03 / 25 / 2009$ & 24.5 \\
& ACIS-S & 9839 & $03 / 25 / 2009$ & 32.0 \\
& ACIS-I & 13574 & $01 / 11 / 2011$ & 2.0 \\
\hline
\end{tabular}

In Sections 2 and 3, we review all the available observational data for CXB3 and CX332 and processes used to examine these data. In Section 4, we examine the light curve for both objects looking for variability within each system. In Section 5, we perform fits to the X-ray spectra of CXB3 with different models in order to identify the type of binary systems. In Section 6, we examine the optical spectra of each candidate optical counterpart star looking for key emission and absorption line features to obtain their spectral type. Finally, we discuss the significance of our results and what they mean for classification of CXB3 and CX332.

\section{DATA PROCESSING}

\subsection{X-ray observations}

A total of 12 different publicly available observations from the $\mathrm{CXO}$ of CXB3 were obtained for analysis. CX332 was observed twice by $\mathrm{CXO}$, once as part of the GBS off-axis by 7.88 arcmin and a second time off-axis by only 0.49 arcmin to obtain a better position. Full analysis of the latter observation is presented in this paper. The details on each observation and their exposure times can be seen in Table 1. All data sets were downloaded from the Chandra Data Archive via Webchaser. Observations were limited to ACIS-I and ACIS-S instruments. The data were reprocessed using Chandra Interactive Analysis of Observations (CIAO) software version 4.9. The events were filtered using an energy range of $0.3-8.0 \mathrm{keV}$ and used $8 \times 8$ binned images as input into wavdetect due to the low numbers of photons detected. While this may introduce some uncertainties in the positions, we believe they are smaller than the systematic uncertainties due to being unable to boresight correct the images. wavdetect was used to measure the position following the methodology in Evans et al. (2010). A weighted average position of CXB3 was also created using all of the extracted positions. With an extraction region established for each observation, light curves were extracted using CIAO's dmextract with $500 \mathrm{~s}$ binning.

Spectra for both targets were also created using the specextract script within CIAO on the same positions used for extracting the lightcurve data. CX332 only had two observations from which to extract any spectra, resulting in a limited number of counts. CXB3 had spectra from 12 different ObsIDs and all were extracted individually. In the last CXO observation of CXB3, the source was found at a higher X-ray luminosity state (see Section 4). In order to compare the target spectra in these two different states, the extracted data of 
the low-luminosity state were summed using the combine_spectra script within CIAO.

XMM-Newton observations of CXB3 from 2010 October 9-10 were also found and obtained from the High Energy Astrophysics Science Archive Research Center (HEASARC). CXB3 was not the primary target and only fell within the field of view of the European Photon Imaging Camera MOS2 detector. The images were processed using Science Analysis System version 18.0.0. Events were processed using EMPROC. Only events with energies $0.3-$ $12.0 \mathrm{keV}$ and PATTERN $\leq 12$ were retained. The source was in a corner of the chip, so rather than a circular region events were extracted from a box rotated to align with the detector to form a spectrum. Consequently, the derived flux may be a mild underestimate of the total, and less reliable than Chandra fluxes. The background spectrum was estimated from a nearby circular region.

\subsection{Optical observations}

The Gemini-South telescope as part of a spectroscopic survey of GBS counterparts observed CXB3 and CX332 with the Gemini Multi-Object Spectrograph (GMOS). A set of two continuous $450 \mathrm{~s}$ exposures for CX332 were obtained on 2018 May 18 using the B1200 grating at a $2.5 \AA$ spectral resolution and a similar set of 570 s exposures for CXB3 were obtained on 2019 April 11 using the R831 grating at $3.4 \AA$ spectral resolution with both using a slit width of 1.0 arcsec. Wavelength coverage for CXB3 spanned 6500$8500 \AA$ while CX332 spanned 6450-8000 ̊. The Gemini IRAF ${ }^{1,2}$ package was used to independently reduce spectra. Flat-fielding used a single flat obtained during the observation runs for each object and wavelength calibration was done following standard GMOS procedures using daytime $\mathrm{CuAr}$ arc spectra. Flux calibration was done using the spectrophotometric standard GD 108. Observations were not taken the same night as the target stars so it will not provide precise calibration but will still remove effects of the instrumental response.

\section{ASTROMETRY}

Localization regions for both SyXB candidates seen in Figs 1 and 2 show improvements over previous positions. Positions and associated errors with these improved positions can be found in Table 2. The second observation of CX332 had significantly fewer counts with the original observation having $\sim 4.0 \times 10^{-3} \mathrm{ct} \mathrm{s}^{-1}$ and the latter observation having $\sim 10^{-4} \mathrm{ct} \mathrm{s}^{-1}$. Despite this, the smaller off-axis angle considerably reduced the size of the error circle from that in the GBS catalogue. The factor of 20 reduction in the area of the error circle, compared to the GBS localization, was sufficient to eliminate most of the alternate counterparts. The new position remains consistent with the carbon star identified by Hynes et al. (2014).

Of the 12 observations for CXB3, there was no significant improvement in the localization region for any single observation. The original estimate was based on an observation that was on-axis with many counts, which left little room for improvement. However, with many different observations of CXB3 combined together into a

\footnotetext{
$1_{\text {WwW.gemini.edu/node/11823 }}$

${ }^{2}$ IRAF is distributed by the National Optical Astronomy Observatories, which are operated by the Association of Universities for Research in Astronomy, Inc., under cooperative agreement with the National Science Foundation.
}

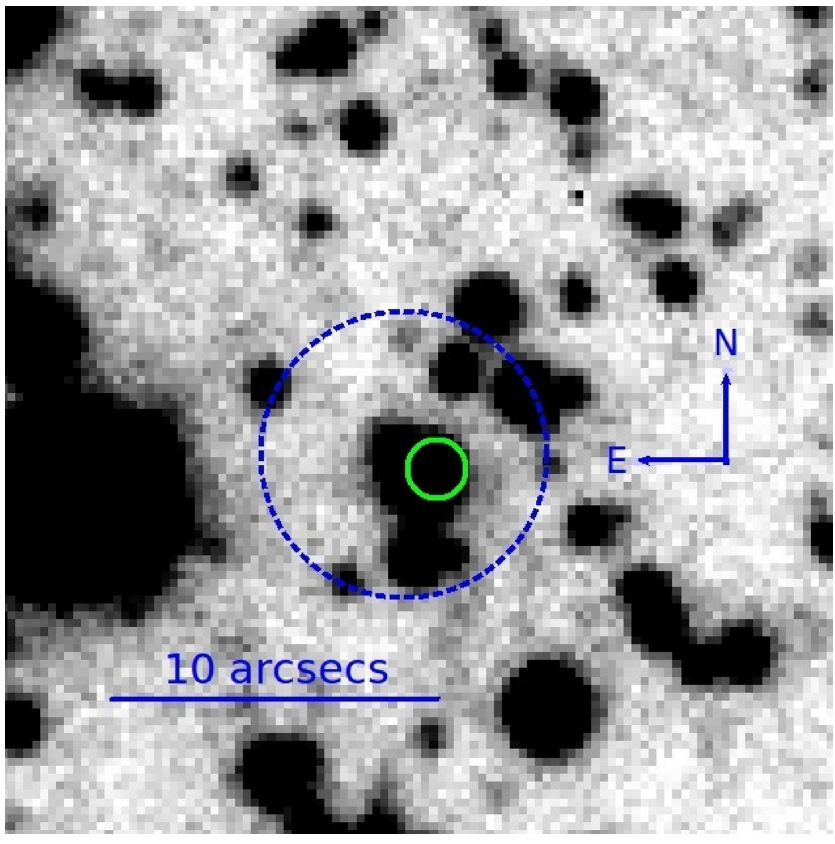

Figure 1. Image of CX332 taken on the Mosaic-2 camera on the Blanco 4-m telescope at CTIO. CX332 is seen highlighted with localized position estimate from the GBS catalogue (dashed) and the new estimate from an observation with an improved off-axis angle (solid). While the new observation had fewer counts, the off-axis angle improvement was able to greatly reduce the uncertainty in the position and eliminate source confusion.

single weighted average, we see 36 per cent improvement over the previous GBS estimate based off a single observation as seen in Table 2 and Fig. 2. We attempted boresight correction of CXB3 by using both the known radio position of H1743-322 and corresponding infrared targets within the same field, but this failed to yield any improvements.

\section{X-RAY SPECTRUM OF CXB3}

An average quiescent spectrum was created by summing together all observations of CXB3 except the high luminosity event observation. The high luminosity event was kept separate to compare the two states. These were made using SHERPA using single count groups for the quiescent state and high-luminosity events. We applied cash statistics to the fitting (Cash 1979).

Thermal plasma and power-law models were applied which have also been used to model WD symbiotic and SyXB systems following Luna et al. (2013) and Masetti et al. (2006). We also examined an observation made with XMM applying the same models. This $86 \mathrm{ks}$ observation was made on 2010 October 9 with ObsID 0553950201 and was processed similarly to the CXO observations with single group counts.

We fit both the quiescent and high-luminosity state of the spectra with the tbabs*powlaw1d power model and separately fitted with the thermal plasma model tbabs*xsapec to compare. All fits to the two states can be seen in Fig. 3. In both cases, tbabs was used to apply an interstellar medium (ISM) absorption model set in SHERPA. ${ }^{3}$ The model parameters and their estimated values for the unabsorbed flux can be seen in Table 3. We quote uncertainties at 90 per cent confidence. An attempt was made to fit the spectra

\footnotetext{
${ }^{3}$ heasarc.gsfc.nasa.gov/xanadu/xspec/manual/XSmodelTbabs.html
} 


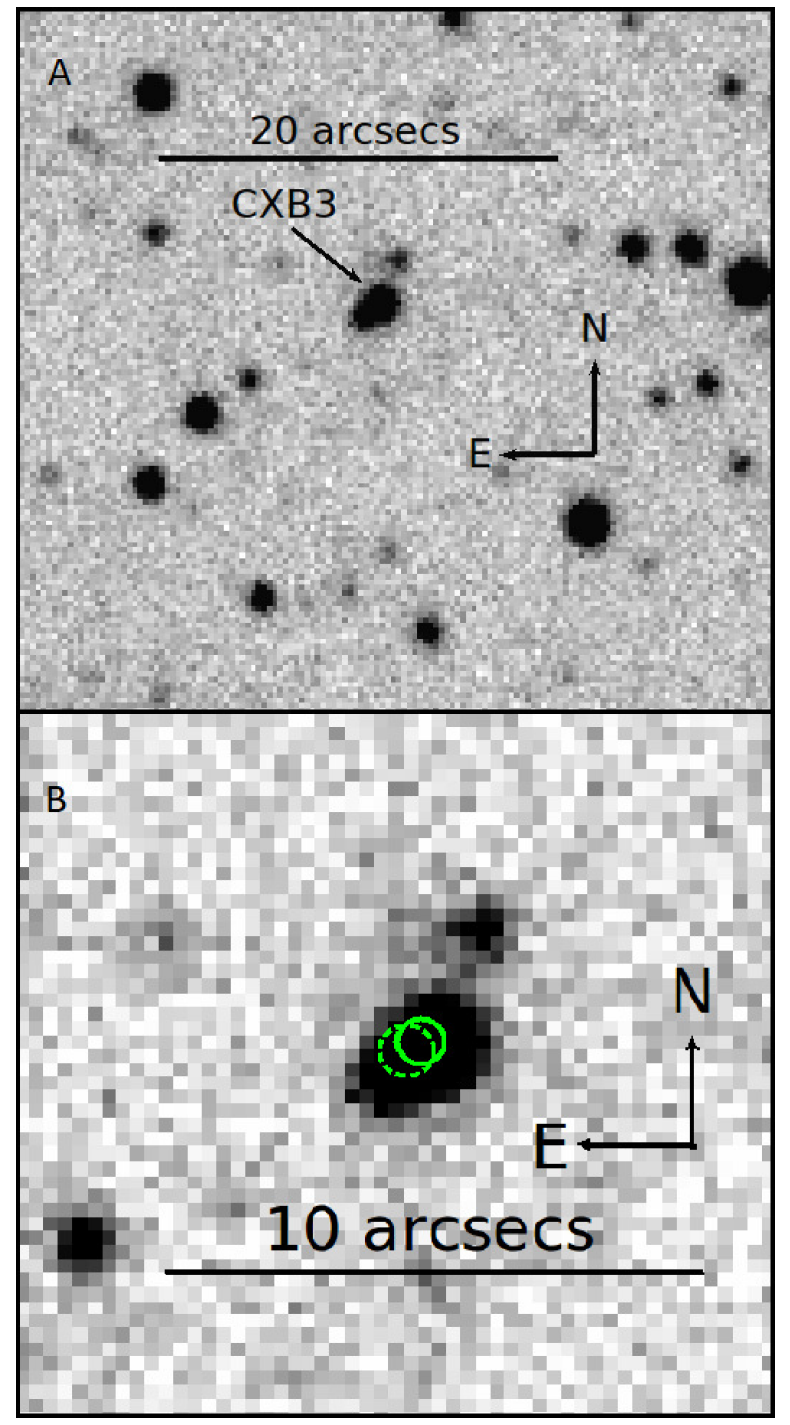

Figure 2. Images of CXB3, the northwestern star in the two star blend, both zoomed out (A) and zoomed in (B) taken with DECam on the Blanco 4-m telescope at CTIO. CXB3 seen highlighted with localized position estimate from the GBS catalogue (dashed) and the new estimate from an observation with an improved off-axis angle (solid).

with a Comptonization model COMPTT, which most commonly is used to fit SyXBs following Masetti et al. (2006, 2007a, b) but fits were unconstrained as the energy range for $\mathrm{kT}$ values reported were beyond CXO's bandpass therefore could not improve upon the power-law model.

The parameters constrained by both models fit well with those seen in other symbiotics and SyXBs fitted with similar models, thus neither model can be strongly preferred. The thermal plasma model best fits a $\delta$-type WD symbiotic star, the most frequent among WD symbiotic star systems (Luna et al. 2013; Nuñez et al. 2014, 2016). The power-law model also was comparable to models fitting $\gamma$-type systems (Muerset et al. 1997; Masetti et al. 2002; Bozzo et al. 2018). We find the XXM spectra are consistent with the quiescent state models from CXO which can be seen in Fig. 4. Unfortunately, the quality of data do not permit robust discrimination between the two models.

\section{LIGHT CURVES}

Light curves in the $0.3-8.0 \mathrm{keV}$ range for CXB3 were compiled and plotted in Fig. 5. The light curve is split into 12 equal time frames from multiple observations over a range of approximately $6000 \mathrm{~d}$. The system was in a low-luminosity quiescent state with approximately constant flux until the last observation, which sees a high-luminosity event with an increase in the brightness by a factor of approximately 6 when compared to the average of the prior observations. This likely indicates an outburst or flare being detected in the system.

The high-luminosity event had an average count rate of $(5.8 \pm 0.8) \times 10^{-2} \mathrm{ct} \mathrm{s}^{-1}$ as compared to the average count rate in the low-luminosity state of $(9.5 \pm 0.2) \times 10^{-3} \mathrm{ct} \mathrm{s}^{-1}$ in previous observation periods. The standard deviation between lowstate observations is $1.7 \times 10^{-3} \mathrm{ct} \mathrm{s}^{-1}$ represents real variability in the system between observations as this variation between individual observations is larger than expected from photon noise. Using model values seen in Table 3 from SHERPA, we estimate an unabsorbed flux of $\left(1.1_{-0.4}^{+0.4}\right) \times 10^{-13} \mathrm{erg} \mathrm{cm}^{-2} \mathrm{~s}^{-1}$ with an unabsorbed luminosity of $\sim 8.4 \times 10^{32} \mathrm{erg} \mathrm{s}^{-1}$ for the low-luminosity state and an unabsorbed flux as high as $\left(2.2_{-1.7}^{+3.9}\right) \times 10^{-12} \mathrm{erg} \mathrm{cm}^{-2} \mathrm{~s}^{-1}$ with an unabsorbed luminosity of $\sim 1.7 \times 10^{34} \mathrm{erg} \mathrm{s}^{-1}$ for the high state, assuming a distance of $d=8 \mathrm{kpc}$. A more careful analysis is done in Section 7 where we find the distance of CXB3 to likely be $8.3-9.5 \mathrm{kpc}$. This luminosity increase is larger than what we see in the light-curve data, which we infer as a larger absorption during the outburst state. It is worth noting that the Swift Bulge Survey did not detect any outburst from CXB3, which in combination with our observations suggests a relatively low duty cycle in the system (Bahramian et al. 2021). We also attempted a search for periodicities in the light-curve data but did not detect any significant periods.

Light-curve data extracted in the $0.3-8.0 \mathrm{keV}$ range for CX332 had counts that were very low compared to CXB3. The count rate from the original GBS observation of CX332 was $(4.0 \pm 1.1) \times 10^{-3}$ $\mathrm{ct} \mathrm{s}^{-1}$ corresponding to an unabsorbed flux of $(9.4 \pm 1.9) \times 10^{-14}$ $\operatorname{erg~cm}{ }^{-2} \mathrm{~s}^{-1}$. The average count rate for the more recent observation was much fainter with $(1.6 \pm 0.8) \times 10^{-4} \mathrm{ct} \mathrm{s}^{-1}$ corresponding to an unabsorbed flux of $(3.9 \pm 0.6) \times 10^{-15} \mathrm{erg} \mathrm{cm}^{-2} \mathrm{~s}^{-1}$. This large dimming demonstrates variability within CX332, which is not atypical behaviour for SyXBs (Bahramian et al. 2014). The corresponding $\mathrm{X}$-ray unabsorbed luminosity values associated with these values using $\mathrm{d}=8.0 \mathrm{kpc}$ are $\sim 7.2 \times 10^{32} \mathrm{erg} \mathrm{s}^{-1}$ for the first observation and $\sim 3.0 \times 10^{31} \mathrm{erg} \mathrm{s}^{-1}$ for the latter. SHERPA with the models used on CXB3 was used to estimate the flux of CX332.

\section{GEMINI-S SPECTROSCOPY}

The full extracted Gemini spectra of the candidate counterparts to CXB3 and CX332 can be seen in Fig. 6. As previously identified by Hynes et al. (2014), CX332 is clearly a carbon star showing multiple CN features with strong bands around 6800-6900 $\AA$ and 7800-7900 $\AA$. There also appears to be a relatively strong Ba II absorption band around $6490 \AA$ suggesting this is a C-N type AGB carbon star, though a $\mathrm{C}-\mathrm{R}$ type still cannot be entirely ruled out as they are difficult to distinguish above $5000 \AA$. There does not appear to be strong evidence of $\mathrm{H} \alpha$ emission that is unusual for a star of this type (Li et al. 2018) or a counterpart in a symbiotic binary, though its absence is common in SyXBs.

CXB3 has a magnitude of $r^{\prime} \sim 20$ (Wevers et al. 2016). Its optical spectrum shows TiO absorption bands around 7100 and $7800 \AA$, which are indicative of an early M-type star. CXB3 only has marginal 
Table 2. Positions with estimated uncertainties derived from each observation.

\begin{tabular}{cccccc}
\hline Object & $\begin{array}{c}\text { RA } \\
\text { (degree) }\end{array}$ & $\begin{array}{c}\text { Dec. } \\
\text { (degree) }\end{array}$ & $\begin{array}{c}\text { Error } \\
(\operatorname{arcsec})\end{array}$ & ObsID & Source \\
\hline CX332 & 264.084324 & -29.560636 & 4.43 & 8693 & GBS \\
& 264.084000 & -29.560757 & 0.92 & 15799 & This Work \\
CXB3 & 266.559988 & -32.330379 & 0.50 & 13574 & GBS \\
& 266.559974 & -32.330082 & 1.41 & 4565 & This Work \\
& 266.559856 & -32.330275 & 0.91 & 4566 & This Work \\
& 266.559832 & -32.330313 & 0.67 & 4567 & This Work \\
& 266.559444 & -32.330143 & 1.73 & 8987 & This Work \\
& 266.559551 & -32.330159 & 2.11 & 8988 & This Work \\
& 266.559796 & -32.330182 & 1.01 & 8989 & This Work \\
& 266.559991 & -32.330473 & 1.21 & 8990 & This Work \\
& 266.560081 & -32.330344 & 1.09 & 9833 & This Work \\
& 266.559721 & -32.330213 & 1.19 & 9837 & This Work \\
& 266.559687 & -32.330196 & 1.00 & 9838 & This Work \\
& 266.560116 & -32.330493 & 0.98 & 9839 & This Work \\
& 266.559965 & -32.330386 & 0.50 & 13574 & This Work \\
& 266.559907 & -32.330334 & 0.44 & Average & This Work \\
\hline
\end{tabular}
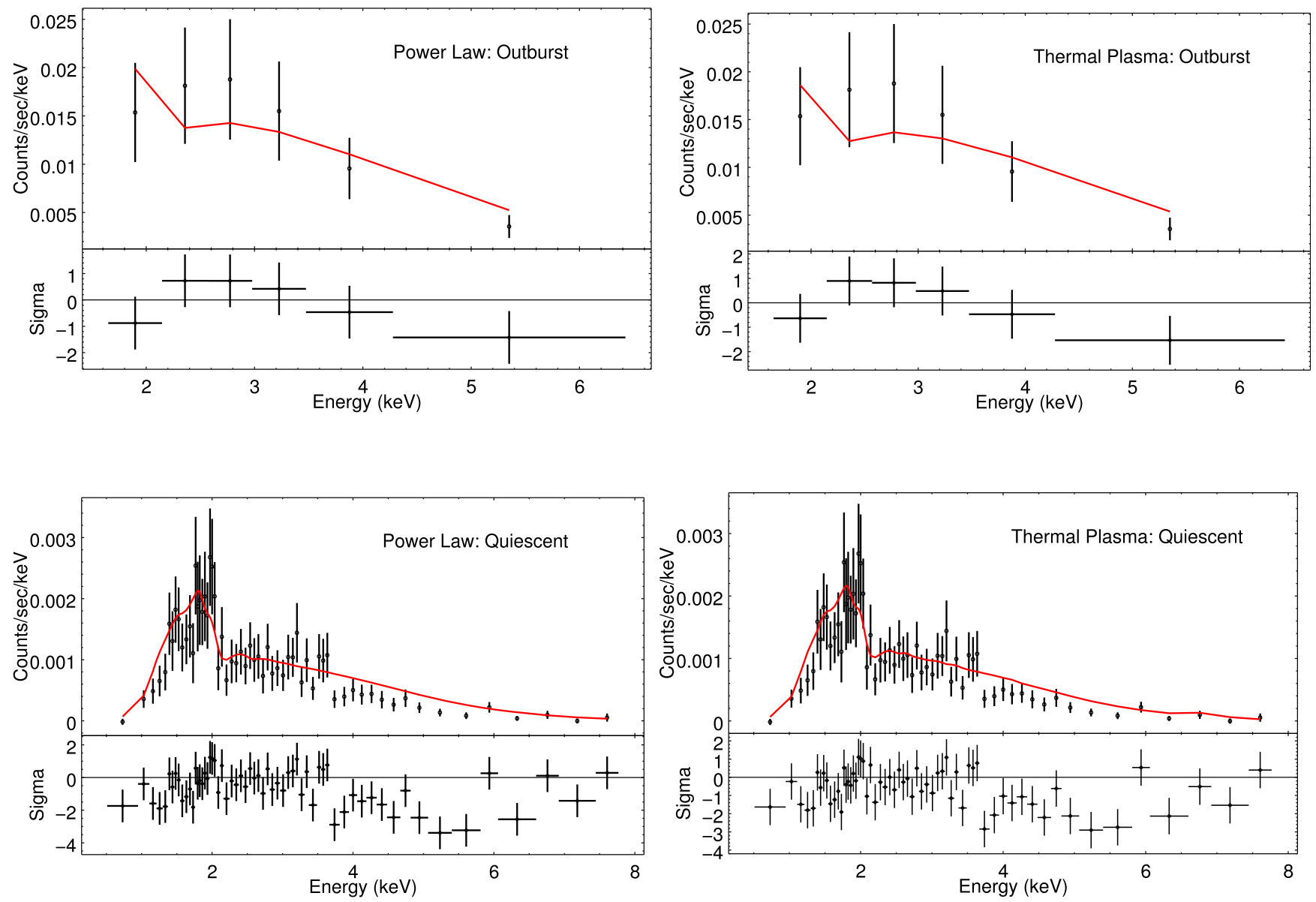

Figure 3. X-ray spectra of CXB3 in both low and high-luminosity states produced using SHERPA. Low-luminosity states were summed together into one long spectrum to increase counts. Error bars are calculated on 15 count bins using chi2gehrels fitting stat that utilizes $\chi^{2}$ statistics with the Gehrels variance function (Gehrels 1986). (Top left) High-luminosity event using tbabs*powlaw1d model. (Top right) High-luminosity event using tbabs*xsapec model. (Bottom left) Low-luminosity state using tbabs*powlaw1d model. (Bottom right) Low-luminosity state using tbabs*xsapec model. 

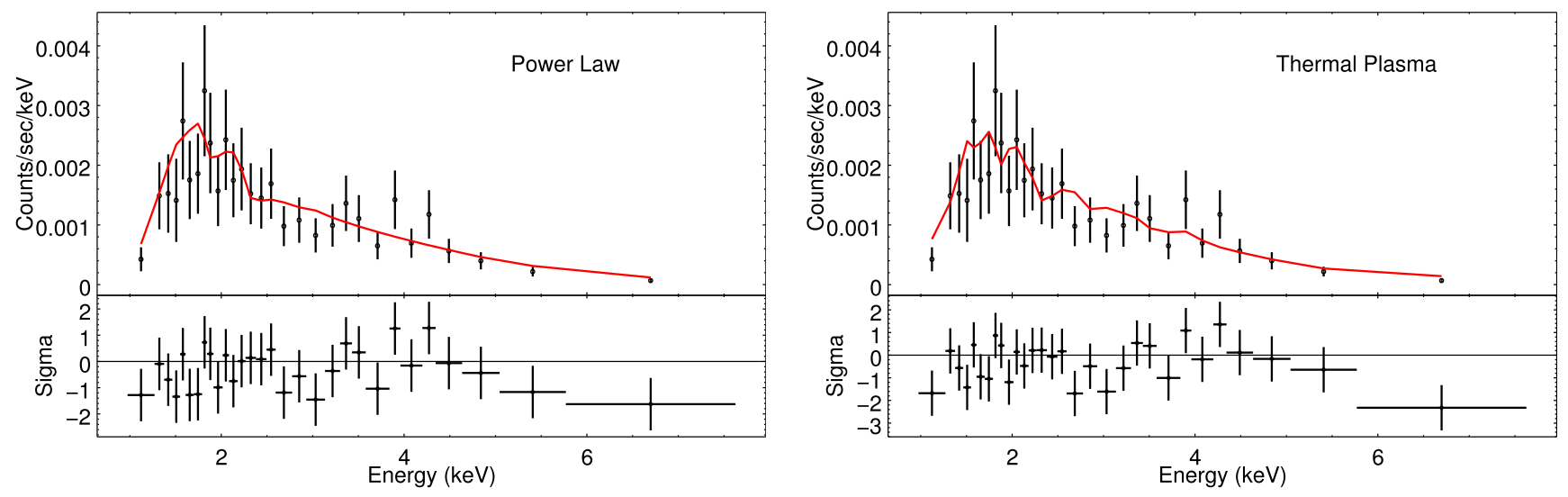

Figure 4. XMM X-ray spectra of CXB3 using SHERPA. The left figure uses the tbabs*powlaw1d model and the right figure uses the tbabs*xsapec model. Error bars are calculated on 15 count bins using chi2gehrels fitting stat that utilizes $\chi^{2}$ statistics with the Gehrels variance function (Gehrels 1986).

Table 3. Parameters for CXB 3 estimated using SHERPA.

\begin{tabular}{|c|c|c|c|c|c|c|c|c|c|}
\hline Detector and State & Model & $\begin{array}{l}\text { Count rate } \\
\left(10^{-3} \mathrm{cps}\right)\end{array}$ & $\begin{array}{c}N_{\mathrm{H}} \\
\left(10^{22} \mathrm{~cm}^{-2}\right)\end{array}$ & $\Gamma$ & $\begin{array}{c}\mathrm{kT} \\
(\mathrm{keV})\end{array}$ & $\underset{\left(10^{-5}\right)}{\operatorname{amp}}$ & $\begin{array}{l}\text { norm } \\
\left(10^{-5}\right)\end{array}$ & $\begin{array}{c}\mathrm{Fx} \\
\left(\mathrm{erg} \mathrm{cm}^{-2} \mathrm{~s}^{-1}\right)\end{array}$ & $\chi^{2}(d o f)$ \\
\hline CXO Quiescence & tbabs $*$ powlaw $1 \mathrm{~d}$ & $9.5 \pm 0.2$ & $1.9_{-0.3}^{+0.3}$ & $1.5_{-0.2}^{+0.2}$ & & $1.6_{-0.4}^{+0.5}$ & & $\left(1.1_{-0.4}^{+0.4}\right) \times 10^{-13}$ & $360(363)$ \\
\hline CXO Quiescence & tbabs $* x s a p e c$ & $9.5 \pm 0.2$ & $1.9_{-0.2}^{+0.2}$ & & $10.6_{-2.9}^{+5.7}$ & & $6.7_{-0.6}^{+0.7}$ & $\left(1.1_{-0.1}^{+0.1}\right) \times 10^{-13}$ & $360(363)$ \\
\hline CXO Outburst & tbabs $*$ xsapec & $57.7 \pm 8.3$ & $2.4_{-0.8}^{+1.1}$ & & $8.3_{-4.9}^{+28.7}$ & & $107_{-23}^{+65}$ & $\left(1.7_{-0.4}^{+0.3}\right) \times 10^{-12}$ & $90(93)$ \\
\hline
\end{tabular}

evidence of $\mathrm{H} \alpha$ emission, which is typical if CXB3 is in fact an SyXB. Magnified view of the $\mathrm{H} \alpha$ region can be as seen in Fig. 6 . The $\mathrm{H} \alpha$ equivalent width is $2.40 \AA$.

\section{DISCUSSION}

The thermal plasma model of CXB3 was most consistent with a WD symbiotic binary system with $\delta$-type X-ray emissions following the schemes in Muerset et al. (1997) and Luna et al. (2013). The estimated X-ray luminosity CXB3 exhibits, however, is not typically seen in wind accreting WD symbiotic systems. The peak X-ray luminosity seen in CXB3 is more likely to be found in SyXBs (Lü et al. 2012; Luna et al. 2013; Nuñez et al. 2014, 2016). The power-law model also cannot be ruled out, which may describe Comptonization taking place in NS SyXBs (Masetti et al. 2007a; Enoto et al. 2014). A powerlaw spectrum emerges in SyXBs due to inverse Compton scattering in hot gas in the vicinity of the neutron star. We attempted to replicate other models used to fit SyXBs (Masetti et al. 2006, 2007a, b) but had little success due to unconstrained temperature. This was most likely due to our inability to detect energies above $10 \mathrm{keV}$. Recent models have also suggested that at low $L_{X}$ wind accretion in SyXBs could fit the thermal plasma model further suggesting this system could still host a neutron star (Yungelson et al. 2019).

We also attempted to compare the extracted spectra with those of magnetic cataclysmic variables (CVs). It remains possible that the detected object in the line of sight of CXB3 is a CV that has a coincidental alignment with the candidate giant counterpart. In that case, it would be expected that CXB3 is much closer than originally estimated, possibly as close as $\sim 5 \mathrm{kpc}$ or even less. Our spectra lacked some characteristic features of magnetic CVs, such as the 6.4-keV Fe emission line with an upper limit of $\sim 3.5 \times 10^{-15} \mathrm{erg} \mathrm{cm}^{-2} \mathrm{~s}^{-1}$ (Balman 2012). It is worth noting that magnetic CVs tend to have exceptionally stable flux and it is not typical to see flux values vary by factors of 30 . While we still can neither confirm nor rule out the possibility of CXB3 being a coincidence of a magnetic $\mathrm{CV}$ with the apparent giant star counterpart, the variable flux and high $L_{\mathrm{X}}$ with an $8 \mathrm{kpc}$ distance make it unlikely.

Our only constraint on the distance to CXB3 comes from priors derived from Galactic structure along the line of sight. We use the Besançon model of stellar population synthesis of the Galaxy (Robin et al. 2003) including updates through version 1612 to construct a population of stars from an area $0.1 \mathrm{deg}^{2}$ centred on CXB3. We include all populations and spectral types but limit the sample to observed $K$ magnitudes of 9.5-10.5, consistent with the 2MASS measurement for CXB3, $K=10.059 \pm 0.038$ (Skrutskie et al. 2006). Most of the objects of this brightness lie in the bulge, and the distance distribution is strongly peaked around $8 \mathrm{kpc}$, although with a foreground tail to the distribution. Formally, this yields a prior on the distance of $8.3_{-2.8}^{+1.6} \mathrm{kpc}$ ( $1 \sigma$ confidence). We do additionally have a colour estimate from 2MASS of $J-K=2.40 \pm 0.06$ (Skrutskie et al. 2006). This is very red, more so than almost all of the objects returned by the simulation, suggesting a distant reddened object. If we additionally constrain the simulation to $J-K>1.5$, then the foreground tail of the distribution disappears and we obtain a distance prior of $8.5 \pm 1.5 \mathrm{kpc}$. Further limiting the colours of the sample to $J-K>2.0$ gives a distance $9.5 \pm 0.9 \mathrm{kpc}$, suggesting an object towards the far side of the bulge. These estimates, while they are uncertain since they are only based on the brightness and colour 

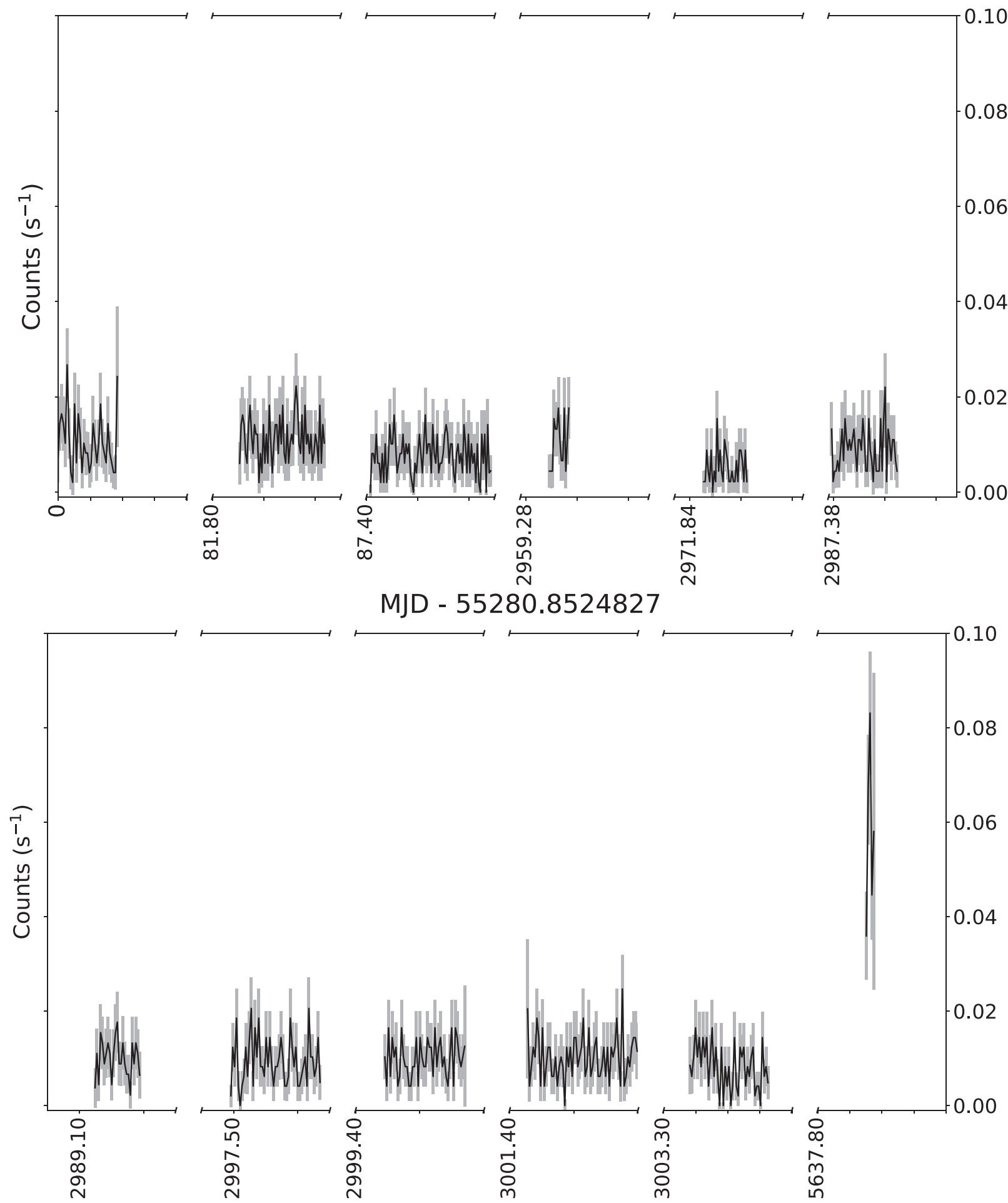

MJD - 55280.8524827

0.08

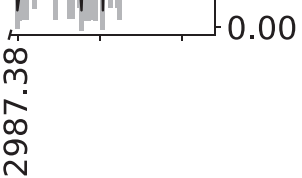

0.06
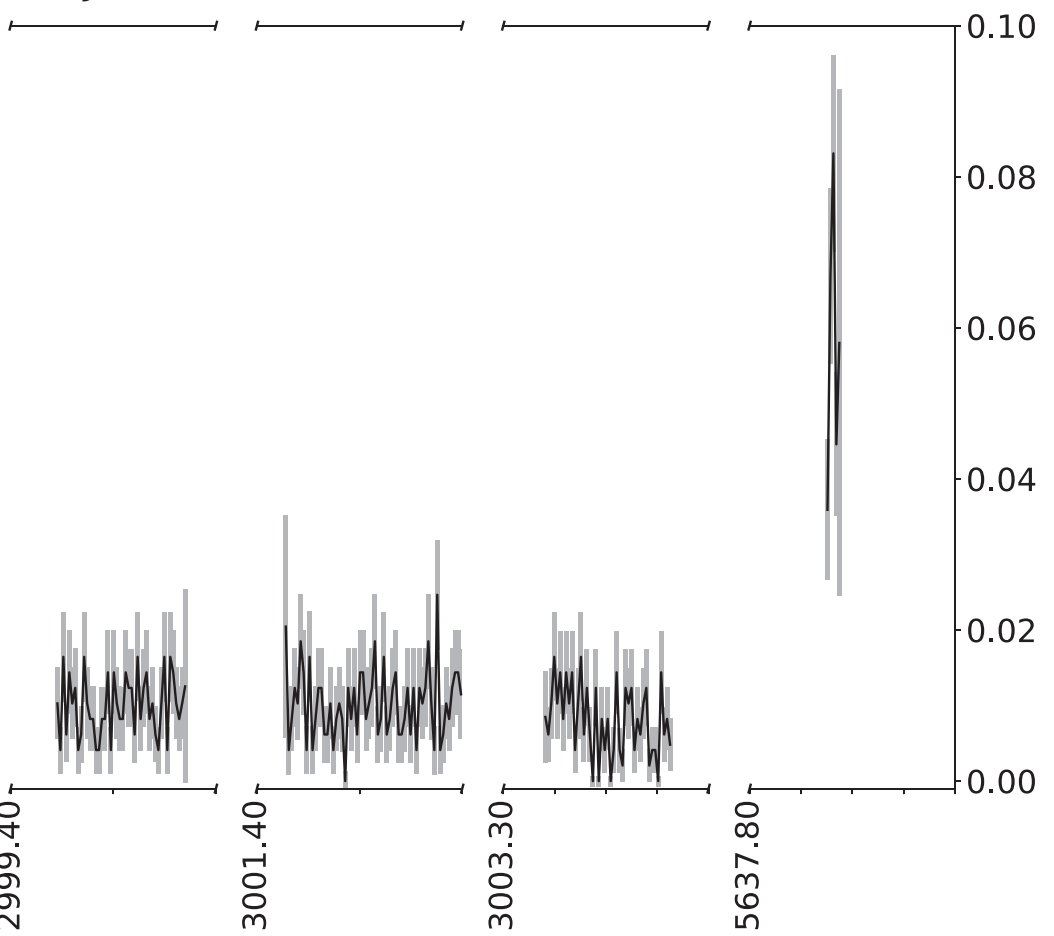

Figure 5. Light curve data of CXB 3 across all observations. Each segment is equally separated into half-day sections. All observations were conducted using the ACIS-S instrument except for the final observation which used the ACIS-I instrument. We estimate the ACIS-I as having an 8 per cent less difference in sensitivity in this observation.

of CXB3, indicate that our assumption of $8 \mathrm{kpc}$ is more likely to be an underestimate than an overestimate. We have also examined Gaia DR2 and EDR3 and concluded that with the large uncertainties in the parallax and inconsistencies between DR2 and EDR3 the data sets are inconclusive at this time, although they are consistent with
CXB3 not being a nearby object (Gaia Collaboration 2016, 2018, 2021).

Assuming a distance of $8 \mathrm{kpc}, \mathrm{CXB} 3$ was captured at a peak luminosity of $L_{X \text {,peak }} \sim 10^{34} \mathrm{erg} \mathrm{s}^{-1}$, though a short exposure makes true peak indeterminable. While WD symbiotics can reach this 

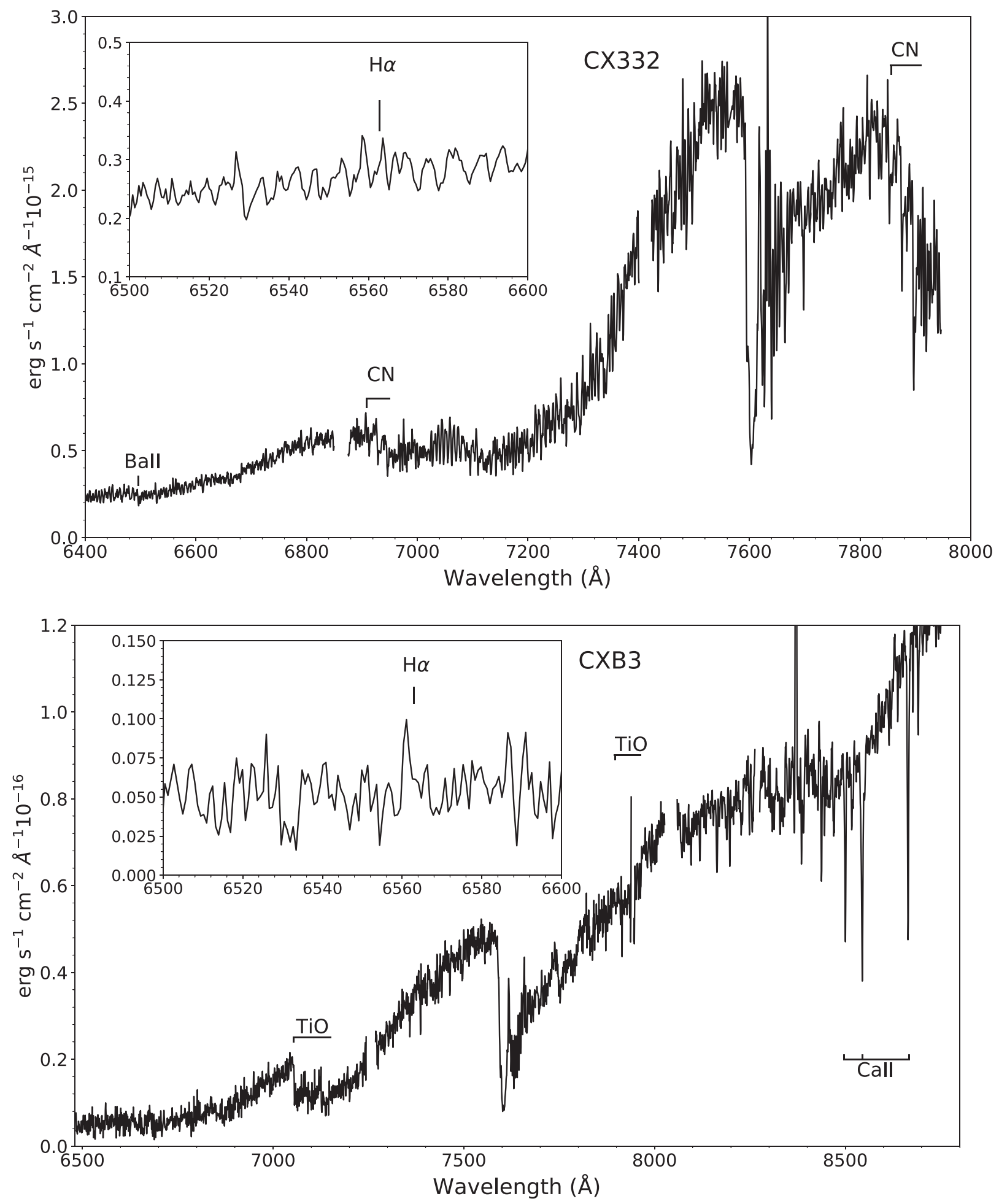

Figure 6. GMOS optical spectrum of CX332 and CXB3.

luminosity (Luna et al. 2018), it is more typically seen in SyXBs. X-ray transients with peak X-ray luminosity $L_{\mathrm{X} \text {,peak }} \sim 10^{34}-10^{36} \mathrm{erg}$ $\mathrm{s}^{-1}$ are not well understood (Degenaar \& Wijnands 2010). Brighter systems are expected to contain either neutron stars or black holes and reach peak X-ray luminosities of $L_{\mathrm{X} \text {,peak }} \sim 10^{36}-10^{39} \mathrm{erg} \mathrm{s}^{-1}$ with well-established temporal and spectral properties related to outbursts caused by a disc instability. This is typically not the case for SyXBs that tend to be dimmer than these values (Lü et al. 2012). While the source GX $1+4$ is an exception with a luminosity of $\sim 10^{37} \mathrm{erg} \mathrm{s}^{-1}$, most of the observed SyXB systems are characterized by a luminosity range $\sim 10^{32}-10^{34} \mathrm{erg} \mathrm{s}^{-1}$ (Masetti et al. 2006) and are highly variable within this range. This is lower than most persistent or outbursting low-mass X-ray binaries (LMXBs) and more typical of quiescent neutron star LMXBs (Reynolds \& Miller 2011). The difference most 
likely results from the fact that accretion on to the compact object takes place via stellar wind rather than via Roche lobe overflow, making the mass transfer process less efficient (Masetti et al. 2011). It is possible that this suppresses the disc instability mechanism that drives LMXB outbursts, or that true outbursts are much rarer than in other well-studied LMXBs. In WD symbiotics, it is also possible that a sufficiently magnetic WD may inhibit formation of a disc entirely. However, due to their long orbital periods, the accretion disc in WD symbiotics should be orders of magnitude larger than those of WD CVs (Duschl 1986; Frank, King \& Raine 2002). This would mean that the magnetic fields in these WD symbiotics would also need to be orders of magnitude in strength compared to those observed in polars or intermediate polars (Cropper 1990; Patterson 1994) to inhibit the formation their accretion disc. There has yet to be any observed symbiotic with such magnetic fields.

We also have GMOS spectra of both targets showing features of an M-type star counterpart for CXB3 and a carbon star for CX332. Neither target had strong evidence of $\mathrm{H} \alpha$ emission lines that are usually present in WD symbiotics and are used as ways to search for them in wide-field $\mathrm{H} \alpha$ imaging (Miszalski, Mikołajewska \& Udalski 2013). Weak or absent $\mathrm{H} \alpha$ emission lines in the optical spectra would instead be expected of SyXBs, which the luminosity of CXB3 would favour. This is likely due to the photoionization by ultraviolet photons from the WD surface. However, it has been suggested that there is a selection effect among WD symbiotics, such as the wide-field $\mathrm{H} \alpha$ emission line searches, that avoids weak-line systems and that these systems may be part of a hidden population that make up a large fraction of total WD symbiotics (Mukai et al. 2016). The weakness or absence of $\mathrm{H} \alpha$ emission might also be expected in accretion-powered WD symbiotics during low-accretion periods. Due to this, we find we cannot rule out either of these systems containing either a WD or neutron star. In either case, these systems are unusual as they could be either hidden WD symbiotics lacking emission lines we would expect in these systems as suggested by Mukai et al. (2016) or SyXBs that fit a thermal plasma model as suggested by Yungelson et al. (2019).

\section{CONCLUSIONS}

We have investigated objects CXB3 and CX332, previously catalogued as candidate symbiotic stars in the GBS. Using data from Chandra, we refined the localization regions of each target. We also used archival observations to examine the spectra and light curves for each object.

CX332 had insufficient data to perform any spectral analysis. We were able to dramatically improve the localization reducing the error circle by a factor of 20 . It remains consistent with a carbon star counterpart though we were not able to conclude anything about its nature as an SyXB candidate (Hynes et al. 2014). The flux of CX332 between the original and the more recent observation showed a change with a ratio between the two being $\sim 25$.

CXB3 was observed as having an increase in brightness in its light curve during the last observational period. The spectrum of CXB3 fits well to a hot plasma model $\delta$-type emission (as also used in identifying WD symbiotics) though recent models suggest an SyXB could also show a thermal spectrum at low $L_{\mathrm{X}}$ (Yungelson et al. 2019). The X-ray luminosity at $8 \mathrm{kpc}$ was most consistent with an SyXB containing a neutron star. Inconclusive data make it difficult to discern whether or not CXB3 could be a symbiotic WD system or a symbiotic binary system containing a neutron star. There remains also the possibility that CXB3 could be a system with a coincidental alignment with a $\mathrm{CV}$ and the distance could also be closer than the $8 \mathrm{kpc}$ assumed when estimating the system luminosity. The GMOS spectrum of CX332 confirms the identity of its counterpart as being a carbon star. The GMOS spectrum of CXB3 indicates its counterpart star as being an M-type giant. In both cases, spectra lacked characteristic features we would typically expect to see in WD symbiotic systems such as $\mathrm{H} \alpha$ emission, though this would be expected in SyXBs as these features are either absent or weak within these systems.

\section{ACKNOWLEDGEMENTS}

Support for this work was provided by the National Aeronautics and Space Administration (NASA) through Chandra Award Numbers GO4-15047X and AR5-16004X issued by the Chandra X-ray Center, which is operated by the Smithsonian Astrophysical Observatory for and on behalf of NASA under contract NAS8-03060. PGJ acknowledges funding from the European Research Council under ERC Consolidation grant agreement number 647208.

The scientific results reported in this article are based on observations made by the Chandra X-ray Observatory as part of proposal \#15400714 and on additional data obtained from the Chandra Data Archive. This research has made use of software provided by the Chandra X-ray Center in the application packages CIAO and SHERPA. This research has also made use of data and software provided by the High Energy Astrophysics Science Archive Research Center, which is a service of the Astrophysics Science Division at NASA/GSFC, in particular for observations obtained with $X M M-$ Newton, an ESA science mission with instruments and contributions directly funded by ESA Member States and NASA.

Gemini observations were obtained under programs GS-2018A-Q314 and GS-2019A-Q-315 at the international Gemini Observatory, a programme of the National Science Foundation's (NSF) NOIRLab, which is managed by the Association of Universities for Research in Astronomy under a cooperative agreement with the NSF on behalf of the Gemini Observatory partnership: the NSF (United States), National Research Council (Canada), Agencia Nacional de Investigación y Desarrollo (Chile), Ministerio de Ciencia, Tecnología e Innovación (Argentina), Ministério da Ciência, Tecnologia, Inovações e Comunicações (Brazil), and Korea Astronomy and Space Science Institute (Republic of Korea).

This work has made use of data from the European Space Agency mission Gaia (https://www.cosmos.esa.int/gaia), processed by the Gaia Data Processing and Analysis Consortium (DPAC, https: //www.cosmos.esa.int/web/gaia/dpac/consortium). Funding for the DPAC has been provided by national institutions, in particular the institutions participating in the Gaia Multilateral Agreement. This publication also makes use of data products from the Two Micron All Sky Survey, which is a joint project of the University of Massachusetts and the Infrared Processing and Analysis Center/California Institute of Technology, funded by NASA and National Science Foundation.

This research has made use of NASA's Astrophysics Data System and the VizieR catalogue access tool, CDS, Strasbourg, France.

We would like to thank the referee for making helpful comments on the manuscript.

\section{DATA AVAILABILITY}

The Chandra data underlying this article are available in the Chandra Data Archive at https://cxc.harvard.edu/cda/ and can be accessed with ObsIDs given in Table 1. XMM-Newton data are available from High Energy Astrophysics Science Archive Research Center at https://heasarc.gsfc.nasa.gov/ and can be accessed with ObsID 
0553950201. Gemini data are available from the Gemini Observatory Archive at https://archive.gemini.edu/searchform and can be accessed by proposal numbers GS-2018A-Q-314 and GS-2019A-Q315 .

\section{REFERENCES}

Bahramian A., Gladstone J. C., Heinke C. O., Wijnands R., Kaur R., Altamirano D., 2014, MNRAS, 441, 640

Bahramian A. et al., 2021, MNRAS, 501, 2790

Balman S., 2012, Mem. Soc. Astron. Ital., 83, 585

Bozzo E. et al., 2018, A\&A, 613, A22

Cash W., 1979, ApJ, 228, 939

Chakrabarty D., Roche P., 1997, ApJ, 489, 254

Corbel S., Kaaret P., Fender R. P., Tzioumis A. K., Tomsick J. A., Orosz J. A., 2005, ApJ, 632, 504

Cropper M., 1990, Space Sci. Rev., 54, 195

Degenaar N., Wijnands R., 2010, A\&A, 524, A69

Duschl W. J., 1986, A\&A, 163, 56

Enoto T. et al., 2014, ApJ, 786, 127

Evans I. N. et al., 2010, ApJS, 189, 37

Frank J., King A., Raine D., 2002, Accretion Power in Astrophysics, 3 edn., Cambridge University Press, Cambridge

Gaia Collaboration, 2016, A\&A, 595, A1

Gaia Collaboration, 2018, A\&A, 616, A1

Gaia Collaboration, 2020, A\&A, 649, A1

Gehrels N., 1986, ApJ, 303, 336

Hynes R. I. et al., 2014, ApJ, 780, 11

Jonker P. G. et al., 2011, ApJS, 194, 18

Jonker P. G. et al., 2014, ApJS, 210, 18

Kenyon S. J., 1986, The Symbiotic Stars. Cambridge Univ. Press, Cambridge, p. 295

Kenyon S. J., Livio M., Mikolajewska J., Tout C. A., 1993, ApJ, 407, L81

Li Y.-B. et al., 2018, ApJS, 234, 31

Lü G.-L., Zhu C.-H., Postnov K. A., Yungelson L. R., Kuranov A. G., Wang N., 2012, MNRAS, 424, 2265
Luna G. J. M., Sokoloski J. L., Mukai K., Nelson T., 2013, A\&A, 559, A6

Luna G. J. M. et al., 2018, A\&A, 616, A53

Maoz D., Mannucci F., Nelemans G., 2014, ARA\&A, 52, 107

Marcu D. M. et al., 2011, ApJ, 742, L11

Masetti N. et al., 2002, A\&A, 382, 104

Masetti N., Orlandini M., Palazzi E., Amati L., Frontera F., 2006, A\&A, 453, 295

Masetti N., Rigon E., Maiorano E., Cusumano G., Palazzi E., Orlandini M., Amati L., Frontera F., 2007a, A\&A, 464, 277

Masetti N. et al., 2007b, A\&A, 470, 331

Masetti N., Munari U., Henden A. A., Page K. L., Osborne J. P., Starrfield S., 2011, A\&A, 534, A89

Mikołajewska J., 2007, Balt. Astron., 16, 1

Miszalski B., Mikołajewska J., Udalski A., 2013, MNRAS, 432, 3186

Muerset U., Wolff B., Jordan S., 1997, A\&A, 319, 201

Mukai K. et al., 2016, MNRAS, 461, L1

Munari U., Renzini A., 1992, ApJ, 397, L87

Nuñez N. E., Luna G. J. M., Pillitteri I., Mukai K., 2014, A\&A, 565, A82

Nuñez N. E., Nelson T., Mukai K., Sokoloski J. L., Luna G. J. M., 2016, ApJ, 824,23

Orio M., Zezas A., Munari U., Siviero A., Tepedelenlioglu E., 2007, ApJ, 661,1105

Patterson J., 1994, PASP, 106, 209

Reynolds M. T., Miller J. M., 2011, ApJ, 734, L17

Robin A. C., Reylé C., Derrière S., Picaud S., 2003, A\&A, 409, 523

Skrutskie M. F. et al., 2006, AJ, 131, 1163

Tutukov A., Yungelson L., 1979, in Conti P. S., De Loore C. W. H., eds, Proc. IAU Symp. 83, Mass Loss and Evolution of O-Type Stars. Reidel, Dordrecht, p. 401

Wevers T. et al., 2016, MNRAS, 458, 4530

Yungelson L. R., Kuranov A. G., Postnov K. A., 2019, MNRAS, 485, 851

This paper has been typeset from a $\mathrm{T}_{\mathrm{E}} \mathrm{X} / \mathrm{L} \mathrm{T}_{\mathrm{E}} \mathrm{X}$ file prepared by the author. 